\title{
Self-insertion of Foreign Objects in Teeth
}

\author{
Bhanu Lakhani ${ }^{1}$, Shalini Garg ${ }^{2}$, Bhavna G Saraf ${ }^{3}$, Ekta Tomer ${ }^{4}$, Nayanika Singh ${ }^{5}$, Neha Sheoran ${ }^{6}$
}

\begin{abstract}
Introduction: Self-insertion of foreign objects in teeth is a common occurrence in children facing dental problems in an attempt to alleviate irritation, drainage, or other symptoms from teeth. However, this could lead to the development of infection and may worsen the condition if not intervened at an early stage. The removal of such objects and the preservation of involved teeth pose a challenge and, in some cases, the tooth must be extracted to resolve the infection.

Aims and objectives: The aim of this article is to report cases of foreign object insertion and discuss the related consequences, thereby, stressing the importance of regular dental visits and maintenance of oral hygiene so as to minimize dental neglect.

Case Studies: The article discusses four case reports of children in the age group 2-14 year old who reported with self-inserted foreign objects in their teeth.

Discussion: Regular dental visits and timely dental management can help reduce the complications associated with such incidents. Ultrasonic instruments, the Masserann kit, periapical surgery and various other techniques have been recommended by the authors for successful removal of foreign objects from the pulp chamber and the canal.
\end{abstract}

Keywords: Foreign object, Hygiene, Neglect, Self-insertion, Teeth.

International Journal of Clinical Pediatric Dentistry (2019): 10.5005/jp-journals-10005-1595

\section{INTRODUCTION}

Injury to both the hard and soft tissues may occur as a consequence of child's habit of placing foreign objects into the mouth. The occurrence of foreign bodies such as metal screws, staple pins, darning needles, pencil leads, beads, and tooth-picks lodged in the exposed pulp chambers of carious or traumatically injured primary and permanent teeth has been reported in the literature. Children use small objects like stapler pin or wire which are easily inserted inside the pulp chambers of fractured teeth to get relief from irritation and feel pleasure during the removal of food particles. Such cases are often diagnosed accidentally on radiographic examination of the tooth. These foreign objects may act as a potential source of infection and may later lead to a painful condition, as sequelae to pulp exposure and lodgment of the foreign body. Detailed case history, clinical, and radiographic examinations are necessary to come to a conclusion about the nature, size, and location of the foreign object. Such cases are an evidence of dental neglect by the parents. The aim of this case series is to highlight and discuss the occurrence of foreign bodies in the oral cavity of children.

\section{Case Description}

The following cases were observed at the Department of Pedodontics and Preventive Dentistry in Sudha Rustagi College of Dental Sciences and Research, Faridabad, between 2015 and 2016.

\section{Case I}

A 2-year-old boy reported with the chief complaint of a plastic bead stuck on the lower front tooth since 2 hours. The mother reported that the patient was playing at home 2 hours back when parents noticed a bead-like object stuck on the lower front tooth due to biting on it. The object did not come out when tried to remove by the hand by the parents. On examination, a red colored, roundshaped bead-like object of a diameter of $6 \mathrm{~mm}$ was seen completely
${ }^{1}$ Department of Pedodontics and Preventive Dentistry, Sudha Rustagi College of Dental Sciences and Research, Faridabad, Haryana, India

${ }^{2}$ Faculty of Pedodontics and Preventive Dentistry, SGT University, Gurugram, Haryana, India

${ }^{3-6}$ Department of Pedodontics and Preventive Dentistry, Sudha Rustagi College of Dental Sciences and Research, Faridabad, Haryana, India

Corresponding Author: Bhanu Lakhani, Department of Pedodontics and Preventive Dentistry, Sudha Rustagi College of Dental Sciences and Research, Faridabad, Haryana, India, Phone: +91 9773810238, e-mail: bhanu.lakhani@gmail.com

How to cite this article: Lakhani B, Garg S, et al. Self-insertion of Foreign Objects in Teeth. Int J Clin Pediatr Dent 2019;12(2):145-149.

Source of support: Nil

Conflict of interest: None

encircling 72 and impinging on marginal gingiva of 72 (Fig. 1A). Diagnosis of the red plastic bead on the 72 was made. The treatment plan involved the removal of foreign body from the crown of 72 . The bead was removed from the tooth using a dental explorer (Fig. 1B). There was minor bleeding from the gingiva during the removal, but there was no report of pain. There is a huge risk of aspiration of such small objects by the child leading to airway obstruction or toxic reactions in case of ingestion. It is, hence, advisable to not give small objects to play to young children and always let them play under parental supervision.

\section{CASE II}

An 8-year-old boy reported with a chief complaint of decayed lower left back tooth since 3 months. The patient reported that a metal ball bearing was embedded in the tooth while he was chewing on it. The patient was asymptomatic. On examination, 75 was carious with a ball bearing lodged in the crown (Fig. 2A). Intraoral periapical radiograph (IOPAR) of 75 revealed that a radiopaque round object was seen extending till the floor of the 

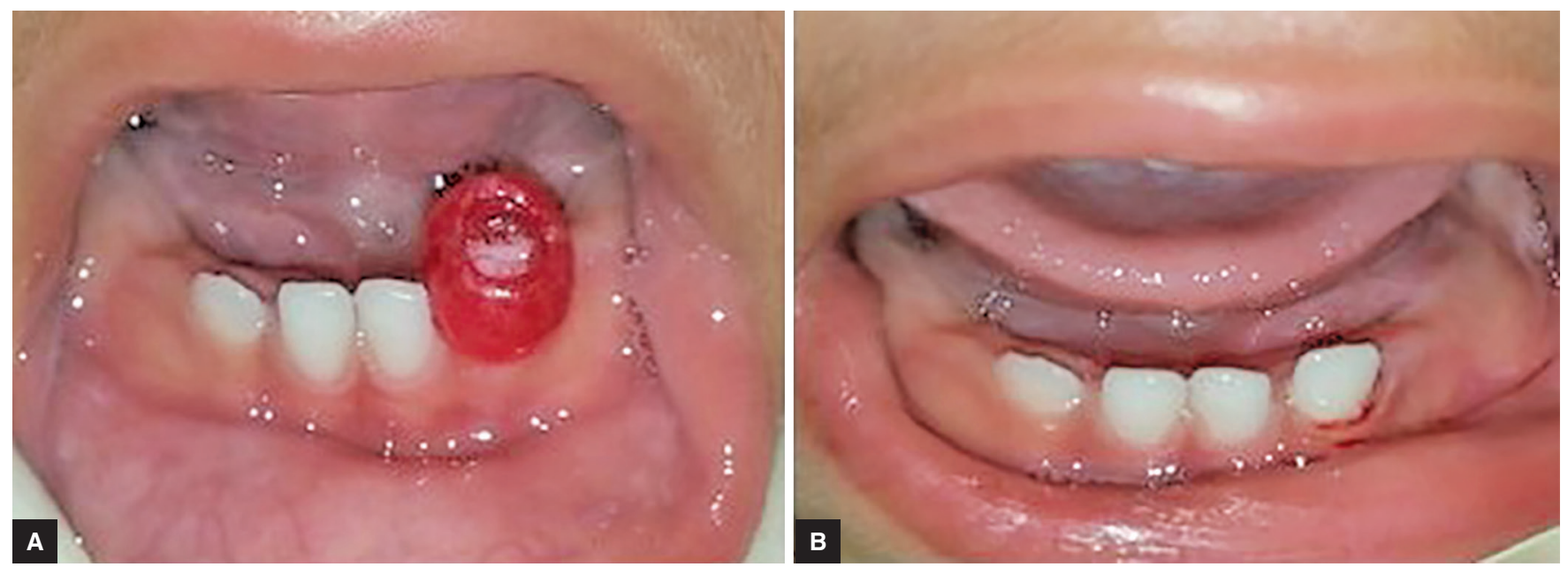

Figs 1 A and B: Case I: A 2-year-old boy reported with a bead stuck on 72, impinging on the gingiva: (A) Plastic bead on 72; (B) After removal of the plastic bead
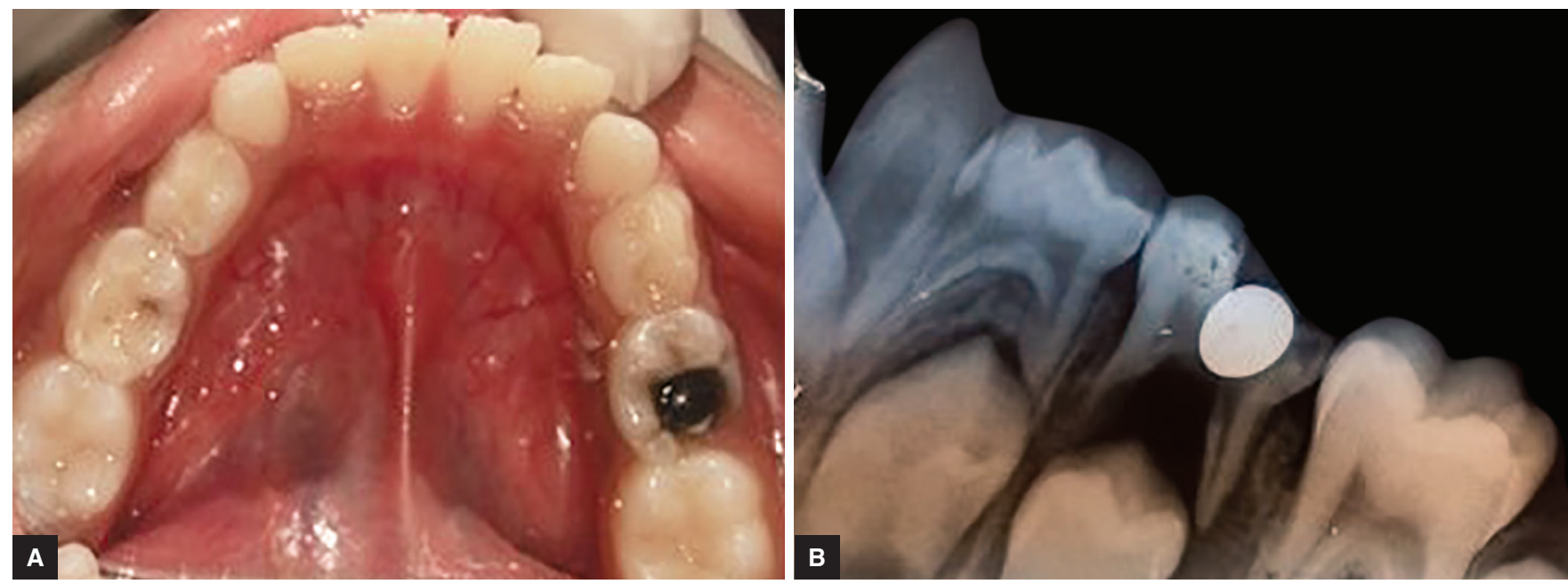

Figs 2A and B: Case II: A 8-year-old boy reported with metal ball bearing embedded in his decayed 75: (A) Ball bearing embedded in decayed 75; (B) Diagnostic IOPAR revealing the involvement of the pulp chamber

pulp chamber (Fig. 2B). External root resorption was evident and furcal radiolucency was observed with 75 . Under local anesthesia, 75 was extracted en mass with the embedded ball bearing. Postextraction healing was satisfactory. The consequences of pushing objects into tooth cavities include the possibility of abscess formation, septicemia, or tetanus. It is, therefore, important to visit the dentist regularly as these cases are a result of dental neglect by the parents.

\section{CASE III}

A 13-year-old male patient reported to the department with a complaint of the broken upper front tooth since a few months. The patient gave a history of trauma to the same tooth while playing kabaddi a few months back. Also complained of recurrent swelling in the palatal mucosa. On clinical examination, the Ellis class III fracture was observed in 21. A diagnostic X-ray revealed large periapical radiolucency with 21 as well as 22 . However, the root canal was radiolucent. Root canal treatment (RCT) of the tooth was planned. After gaining access into the root canal, some resistance was felt on filing. On further circumferential filing with
$\mathrm{H}$ files, multiple broke pieces of matchsticks were recovered from the canal. The patient admitted to have inserted matchsticks in the tooth whenever he experienced pain. All the pieces were removed and the canal was irrigated copiously. The patient was given longterm calcium hydroxide dressings for 2 months to allow healing of periapical changes. The tooth was later obturated with gutta-percha. The polycarbonate crown was cemented and the patient kept on follow-up. There is a huge possibility of development of nonhealing pathologies and formation of granuloma in long-standing cases. It is, therefore, advisable to not to ignore dental problems and encourage the child to maintain oral hygiene (Fig. 3).

\section{Case IV}

A 14-year-male patient reported with the chief complaint of the broken upper front tooth since a few years. The patient gave a history of trauma to the same tooth due to fall on the floor. On clinical examination, the Ellis class III fracture was seen in 21 and the Ellis class II fracture was seen in 11 (Fig. 4A). Diagnostic IOPAR revealed periapical radiolucency with 21 with immature root formation. Root canal treatment was planned for 21 and restoration 

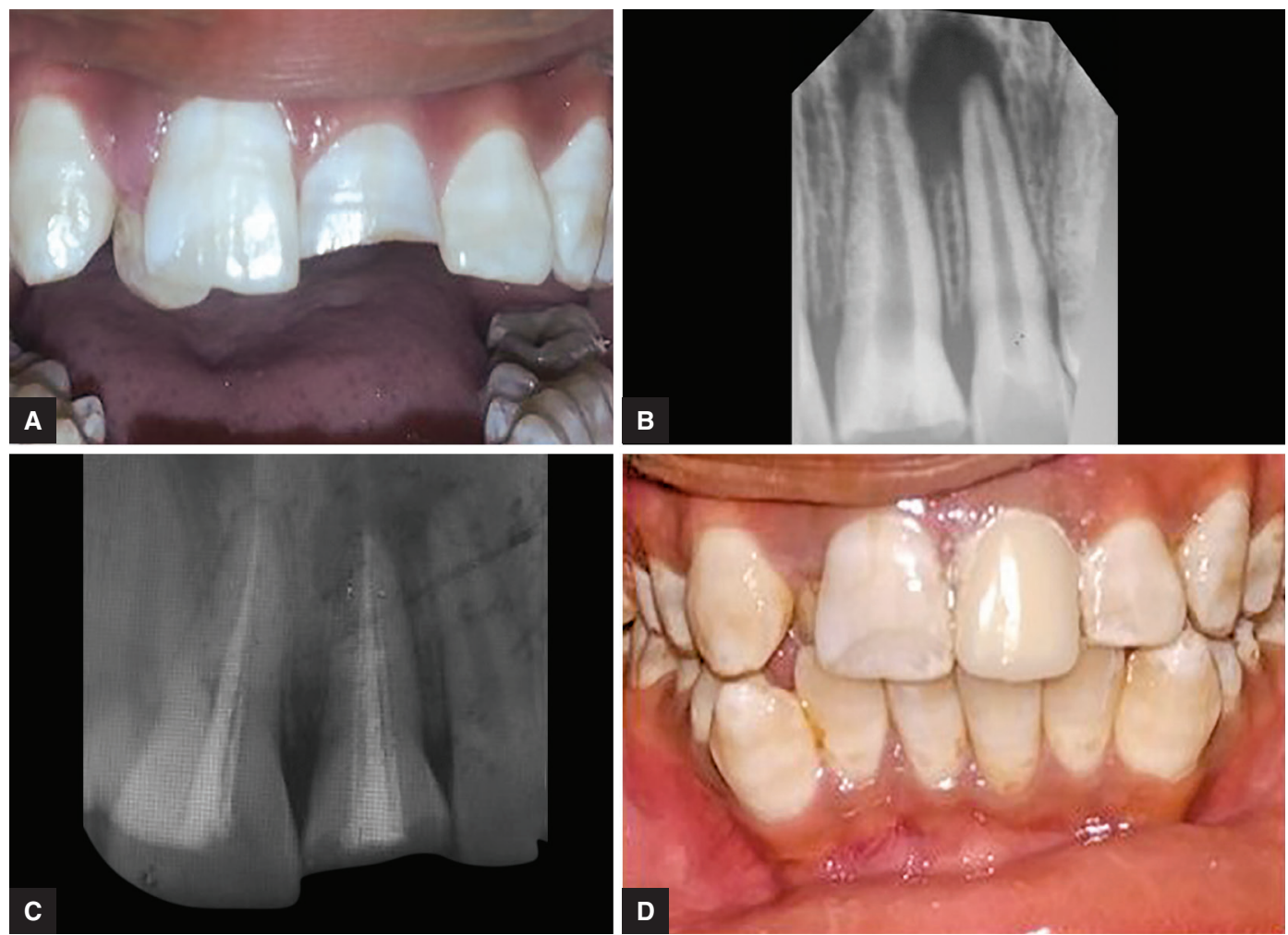

Figs 3A to D: Case III: A 13-year-old boy reported with the Ellis class III fractured 21 with a history recurrent swelling and pain: (A) The Ellis class III fractured 21; (B) Diagnostic IOPAR showing periapical radiolucency with 11 and 21; (C) Obturation after the resolution of infection in 11 and 21 ; (D) Polycarbonate crown cemented on 21
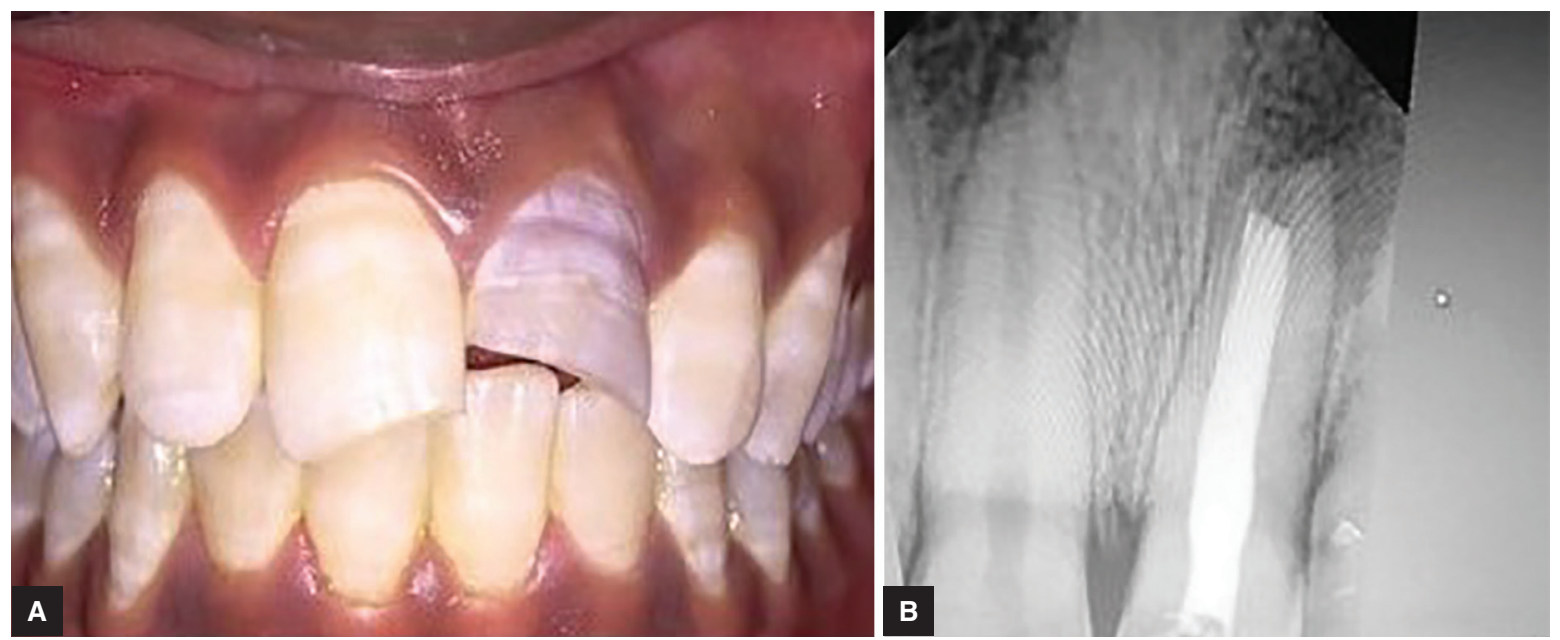

Figs 4A and B: Case IV: A 14-year-old boy reported with the Ellis class III fractured 21: (A) The Ellis class fractured 21; (B) Metapex obturation

was planned for 11. After gaining access into the root canal of 21, a matchstick was recovered from the canal, which was removed and the canal was irrigated copiously. The patient was given multiple dressing and obturated on a subsequent visit with metapex to allow healing of periapical changes (Fig. 4B). Dental neglect is the root cause for foreign object insertion by the child in an effort to reduce the symptoms. However, this could lead to foreign body reactions or abscess formation. Thus, timely dental management and regular dental visits are a must to avoid such situations (Fig. 5).

\section{Discussion}

Children are inquisitive by nature and as part of their development, they explore their surroundings with the use of tactile sensation. Injury to both the hard and soft tissues may occur as a consequence of child's habit of placing foreign objects into the mouth. Such behavior is also possible when pulp chambers are exposed to the oral cavity either as traumatic injuries/carious exposure or left opened for drainage during the root canal procedure. Children use foreign objects that can be easily inserted and are readily available 

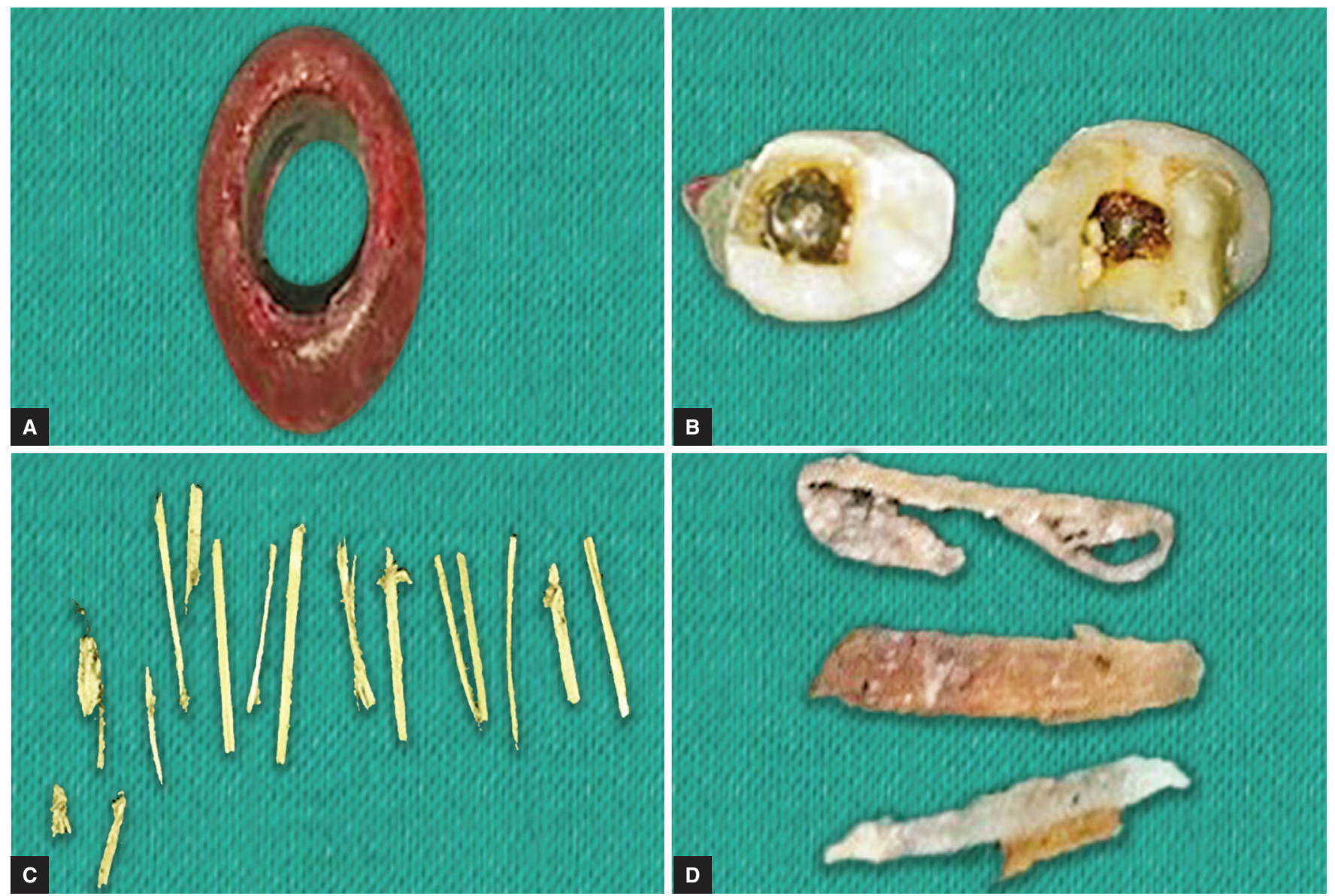

Figs 5A to D: Foreign objects: (A) Plastic bead; (B) Metal ball bearing; (C) Matchsticks; (D) Stapler pins and sticks

such as toothpicks, stapler pins, or wires to remove food particles entrapped inside the open pulp chambers of fractured teeth. These acts provide relief from irritation and pleasure during the removal of food particles. The child knows about the act but never expresses it to the parents until and unless consequential complications like pain develop.' Dental neglect on the part of parents causes the children to resort to such measures as well as leads to a long-term sequelae before the foreign body is discovered.

Foreign bodies lodged in soft tissues like gingiva or soft palate may cause inflammation and scarring. The consequences of implanted/embedded intraoral foreign bodies include infections, pain or abscess, cyst, swelling, and tumor-like appearance. ${ }^{2}$ Reports of chronic maxillary sinusitis and actinomycosis due to intraoral foreign bodies exist in the literature. ${ }^{3}$ Foreign body in deciduous dentition can lead to perforation of the pulp chamber floor space and possibly interfering with developing permanent dentition in the form of odontoma, impaction, and dilaceration. ${ }^{2}$ The possibility of foreign body reaction, formation of granuloma, and non-healing pathology may develop over a long period of time in case of no intervention.

There is also a potential risk of smaller items being ingested or, more seriously, aspirated leading to the obstruction of the airway. Ingested metal objects may be toxic or may generate toxic chemicals from reactions with body fluids and acids. Most foreign body aspirations occur in children younger than 5 years old, with $65 \%$ of deaths affecting infants younger than 1 year.

Azodo et al. in 2015 reported a similar case to case I wherein a 6-year-old child was habitually chewing on biro and a cutoff piece of the inner tube was embedded on $72 .{ }^{4}$ Harris reported a case of an 11-year-old girl with pain in the right central incisor along with soft swelling of the gingiva and a large periapical lesion similar to case III. Wooden pieces from toothpicks and matchsticks were recovered from the root canal among several other objects. $^{5}$

A radiograph can be of diagnostic significance especially if the foreign body is radiopaque. Hunter and Taljanovic ${ }^{6}$ summarized various radiographic methods to be followed to localize a radiopaque foreign object as parallax views, vertex occlusal views, triangulation techniques, stereo radiography, and tomography.

Ultrasonic instruments, the Masserann kit, modified Castroveijo needle holders, ethylenediaminetetraacetic acid, which acts to lubricate the canal, have been recommended by the authors for successful removal of foreign objects from the pulp chamber and the canal. Periapical surgery or intentional reimplantation has also been suggested to remove foreign objects. It has also been reported that removal of a small amount of tooth structure facilitates removal of objects embedded in the tooth. ${ }^{7}$

Literature review has reported that chances of discovering foreign objects in the oral cavity are comparatively higher in children than in adults. This warrants the need for spreading dental awareness and emphasizing the importance of early dental visits and establishing a dental HOME. Such measures will lead to early diagnosis and prompt treatment, hence, minimizing the possible complications. 


\section{References}

1. Srivastava VK. Self-inserted foreign objects inside root canal of fractured incisors: an unusual behaviour. J Indian Dent Assoc 2014 Aug;8(8):31-35.

2. Katge $F$, Mithiborwala S, et al. Incidental radiographic discovery of a screw in a primary molar: an unusual case report in a 6 year old child. Case Rep Dent 2013;2013:296425. DOI: 10.1155/2013/296425.

3. Goldstein BH, Sciubba JJ, et al. Actinomycosis of the maxilla: review of literature and report of case. J Oral Surg 1972;30:362-366.
4. Azodo CC, Erhabor $\mathrm{P}$, et al. Intraoral foreign body: A case report and review of literature. Indian J Multidiscip Dent 2015 Jul 1;5(2): 97.

5. Harris WE. Foreign bodies in root canals: report of two cases. J Am Dent Assoc 1972 Oct 1;85(4):906-911.

6. Hunter TB, Taljanovic MS. Foreign Bodies 1. Radiographics 2003 May;23(3):731-757. DOI: 10.1148/rg.233025137.

7. Aduri $\mathrm{R}$, Reddy $\mathrm{RE}$, et al. Foreign objects in teeth: retrieval and management. J Indian Soc Pedod Prev Dent 2009 Jul 1;27(3):179. DOI: 10.4103/0970-4388.57100. 